\title{
Comparação de diferentes diluentes na mensuração da concentração espermática de suínos em espectrofotômetro
}

\section{Comparison of different diluents to measure swine sperm concentration on the spectrophotometer}

\author{
Carine Dahl Corcini ${ }^{1 *}$; Antonio Sergio Varela Junior²; Rudy Pigozzo ${ }^{3}$; Denise \\ Calisto Bongalhardo ${ }^{1}$; Thomaz Lucia Junior ${ }^{1}$
}

\begin{abstract}
Resumo
Para obtenção de sucesso em um programa de inseminação artificial (IA) é fundamental que as centrais de IA produzam doses inseminantes (DI) de alta qualidade, um fator importante é a correta concentração de espermatozóides por DI. Para a determinação da concentração espermática pode se utilizar o espectrofotômetro. Esta técnica consiste na leitura da concentração espermática através do grau de transmitância da luz na amostra do ejaculado. Geralmente para a execução desta técnica são utilizadas soluções cancerígenas, como a formalina, ou neurotóxicas, como o glutaraldeído. Este trabalho objetivou comparar diferentes diluentes para a mensuração da concentração espermática de suínos no espectrofotômetro. Foram utilizados 53 machos reprodutores suínos de diferentes linhagens e com fertilidade conhecida. O ejaculado foi colhido através do método da mão enluvada. Foi utilizado um aparelho de espectrofotometria (QUIMIS Q-798DMR), previamente calibrado com o sêmen dos animais da própria granja. Nas cubetas foram adicionados 2,97 mL de formol salina (T1) ou soro fisiológico (T2) e acrescido de $0,03 \mathrm{~mL}$ da amostra do ejaculado, sendo a leitura efetuada no visor do aparelho. Os dados foram analisados utilizando ANOVA do software Statistix 2003. Não houve diferença entre os

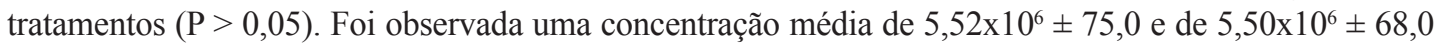
espermatozóides por $\mathrm{mL}$, que corresponde a uma transmitância de $68,2 \pm 2,5$ e $67,1 \pm 2,5$, para T1 e T2 respectivamente. A substituição das soluções não acarreta perdas no resultado obtido e permite que se mantenha um laboratório livre de formol. Portanto, a utilização de soro fisiológico na leitura de ejaculados no espectrofotômetro é o mais indicado para centrais de IA em suínos.
\end{abstract}

Palavaras-chave: Sêmen, suíno, espectrofotômetro, solução diluídora

\begin{abstract}
To obtain success in an artificial insemination (AI) program, it is essential that the AI centrals produce high quality insemination doses (ID); one important factor is to use the correct sperm concentration by ID. To determine sperm concentration, spectrophotometry could be used. This technique consists in the reading of sperm concentration through the measurement of light transmittance percent in the ejaculate sample. Usually, when executing this technique, cancerigenous solutions, as formalin, or neurotoxic solutions, as glutaraldehyde, are used. This work aimed to compare different diluent solutions to measure swine sperm concentration on the spectrophotometer. Fifty three swine male breeders from different strains and with proven fertility were used. Ejaculates were collected by the gloved hand method. A spectrophotometer (QUIMIS Q-798DMR), previously calibrated with the semen from the
\end{abstract}

\footnotetext{
1 Profs. da Universidade Federal de Pelotas, UFPeL, Pelotas, RS. E-mail: corcinicd@gmail.com; denisecb@ufpel.edu.br; thomaz@pq.cnpq.br

2 Prof. da Universidade Federal de Rio Grande, FURG, Rio Grande, RS. E-mail: antoniovarela@furg.br

3 Discente da UFPeL, Pelotas, RS. E-mail: rudypigozzo@yahoo.com.br

* Autor para correspondência
}

Recebido para publicação 06/08/2010 Aprovado em 19/10/2011 
animals of the own farm, was used. In the cuvettes, $0.03 \mathrm{~mL}$ of the ejaculate sample was added to 2.97 $\mathrm{mL}$ of saline phormol (T1) or physiologic solution (T2). Data was analyzed by ANOVA using Statistix

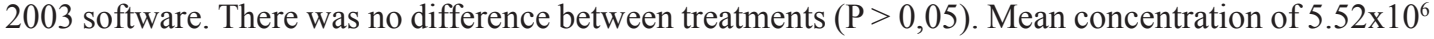
\pm 75.0 and $5.50 \times 10^{6} \pm 68.0 \mathrm{sperm} / \mathrm{mL}$, corresponding to $68.2 \pm 2.5$ and $67.1 \pm 2.5 \%$ transmittance were observed for T1 and T2, respectively. Replacing saline formalin with physiologic solution does not bring losses in the results and keeps the laboratory formalin-free. Therefore, using physiologic solution to read ejaculate concentration on the spectrophotometer is more indicated in swine AI centrals.

Key words: Semen, swine, spectrophotometer, diluent solution

Para que um programa de inseminação artificial (IA) tenha sucesso um dos fatores que se deve atentar é a concentração do ejaculado do macho destinado a produção de doses inseminantes. É importante que a mensuração seja determinada precisa e rapidamente, pois estimativa incorreta da concentração espermática pode ser a causa de problemas de infertilidade, com prejuízos na produtividade e aumento do custo de produção das doses (HANDELSMAN, 2002; WABERSKI; PETRUNKINA; TÖPFER-PETERSEN, 2008).

A concentração espermática deve ser determinada de forma padronizada, podendo ser realizada utilizando o espectrofotômetro que é uma técnica menos subjetiva do que a câmara de Neubauer (PAULENZ et al., 1995). Assim como na execução da leitura na câmara de Neubauer, no espectrofotômetro também é utilizada solução diluidora para leitura contendo formalina (formolsalina, formol-citrato) (ARTEGA; BARACALDO; BARTH, 2001; PRATHALINGAM et al., 2006), glutaraldeído (SCHMIDT-HEBBEL et al., 2000) ou Dodecil sulfato de sódio (PRATHALINGAM et al., 2006). Estas substãncias apresentam característica espermicida necessitando que o técnico evite o contato com o material de processamento de sêmen na central de IA para não contaminar e assim prejudicar a qualidade das doses produzidas. A utilização dessas substâncias formalina que é carcinogênica, glutaraldeído que é neurotóxico também podem causar prejuízo na saúde do técnico responsável pela execução da análise. Portanto a busca de alternativas de diluidor para leitura sem a característica espermicida ou tóxica ao ser humano deve ser testada. Este trabalho objetivou comparar diferentes diluentes de mensuração da concentração em espectrofotômetro de ejaculado de suínos.

O experimento foi realizado em uma Central de Inseminação artificial localizado no interior do RS, onde foram utilizados 53 machos reprodutores suínos de diferentes linhagens. Todos os machos foram manejados sob as mesmas condições ambientais, sendo arraçoados duas vezes ao dia. Foram realizadas duas coletas de cada macho, totalizando 106 ejaculados, durante um período de 15 dias. Os ejaculados foram coletados pelo método da mão enluvada, utilizando frascos aquecidos a $36^{\circ}$ C e cobertos com filtro, a fim de separar a fração gelatinosa do sêmen (HANCOCK; HOVEL, 1959). Em todo o ejaculado coletado, foram mensurados o volume $(\mathrm{mL})$ e a concentração espermática pela técnica de espectrofotometria (QUIMIS Q-798DMR). Para análise de concentração foram utilizados dois meios: controle formol salina (T1) e soro fisiológico (T2). Foi utilizado $2,97 \mathrm{~mL}$ do diluídor e $0,03 \mathrm{~mL}$ da amostra do ejaculado, sendo a leitura realizada no visor do aparelho. $\mathrm{O}$ aparelho foi calibrado utilizando os mesmos animais em pouco tempo antes da leitura. A variável foi avaliada pelo teste de normalidade Shapiro-Wilk. De acordo com este teste, a mesma apresentou distribuição normal $(\mathrm{P}>0,05)$. A análise foi feita através de análise de variância convencional, as comparações entre médias foram realizadas através do teste de Tukey. Todas as análises estatísticas foram conduzidas através do software Statistix 8.0® (2003).

A média da concentração espermática ( \pm erro padrão) foi de $3,82 \times 10^{6} \pm 76,0$ espermatozóides 
por $\mathrm{mL}$. $\mathrm{O}$ volume médio ( \pm erro padrão) dos $10^{6}$ ejaculados foi de $373 \pm 228,79 \mathrm{~mL}$. Quando se utilizou o T1 se obteve uma concentração média de $5,52 \times 10^{6} \pm 75,0$ espermatozóides por $\mathrm{mL}$ que corresponde a uma transmitância de 68,2 $\pm 2,5$ não diferindo $(\mathrm{P}<0,05)$ do $\mathrm{T} 2$ com concentração média de espermatozóide por $\mathrm{mL}$ de $5,50 \times 10^{6} \pm 68,0$ e uma transmitância de 67,1 $\pm 2,5$.

A Agência Nacional de Vigilância Sanitária (ANVISA, 2008) regulamenta quea concentração máxima de formol nas soluções está limitada a $0,5 \%$ desde setembro de 2004. Conforme a Monografia $n^{\circ} 88$ publicada pela IARC (Agência Internacional de Pesquisa do Câncer) em 2006 esta substância é classificada como carcinogênica (causador de câncer). A formulação do formol salina que é utilizado para fazer as análises de concentração das amostras de sêmen possui uma concentração de 5\% sendo 10 vezes superior ao nível de segurança estabelecido pela ANVISA.

A Câmara de Neubauer tem como desvantagem maior demanda no tempo de análise, além de que essa técnica que associam uma menor precisão ao aumento da diluição, pois há sobreposição e desuniformidade ao se preencher a câmara. A distribuição dos espermatozóides pela câmara é, portanto, importante e deve ser heterogênea (PAULENZ et al., 1995). O espectrofotômetro é um método mais rápido que o da Câmara de Neubauer, otimizando o tempo do processamento do sêmen. Desta forma faz com que no período de uma hora seja processado um maior número de ejaculados.

Além de que o custo para produção de $1 \mathrm{~L}$ de formol-salina é 5 vezes maior que de $1 \mathrm{~L}$ solução salina, desta forma além de diminuir o custo também beneficia a saúde do operador e evita a contaminação do ambiente.

Devido à ausência de diferença entre os tratamentos utilizados neste experimento a utilização de solução salina é o mais indicado para a avaliação de concentração no espectrofotômetro de sêmen suíno.

\section{Referências}

ANVISA. Agência Nacional de Vigilância Sanitária. Dispõe sobre conservantes permitidos para produtos saneantes. RESOLUÇÃO-RDC No- 35, de 3 de junho de 2008, Artigo 4. 2008.

ARTEGA, A.; BARACALDO, M.; BARTH, A. D. The proportion of beef bulls in western Canada with mature spermiograms at 11 to 15 months of age. Can. Vet. J., v. 42, n. 10 , p. 783-787. 2001.

HANCOCK, J. L.; HOVELL, G. J. R. The collection of boar semen. Veterinary Record, v. 71, p. 664-665, 1959.

HANDELSMAN, D. J. Optimal power transformations for analysis of sperm concentration and other semen variables. J Androl, v. 23, n. 5, p. 629-34, 2002.

IARCMONOGRAPHS. Formaldehyde, 2-Butoxyethanol and 1-tert-Butoxypropan-2-ol. 2006. v. 88. Disponível em: <http://monographs.iarc.fr/ENG/Monographs/vol88/ mono88-6.pdf>. Acesso em: 19 out. 2011.

PAULENZ, H.; GREVLE, I. S.; TVERDAL, A.; HOFMO, P. O.; BERG, K. A. Precision of the Coulter ${ }^{\circledR}$ for routine assessment of boar-sperm concentration in comparison with the haemocytometer and spectrophotometer. Reproduction in Domestic Animals, v. 30, n. 3, p. 107111, 1995.

PRATHALINGAM, N. S.; HOLT, W. W.; REVELL, S. G.; JONES, S.; WATSON, P. F. The precision and accuracy of six different methods to determine sperm concentration. Journal of Andrology, v. 27, n. 2, p. 257262, 2006.

SCHMIDT-HEBBEL, J.; TONIOLLO, G. H.; LEITE, F. G.; FERRAUDO, A. S.; PERECIN, D.; PACOLA, L. J. Características físicas e morfológicas de sêmen de touros jovens das raças Gir, Guzerá, Nelore (Bos taurus indicus) e Caracu (Bos taurus taurus). Arquivo Brasileiro de Medicina Veterinária e Zootecnia, Belo Horizonte, v. 52, n. 5, p. 461-467, 2000.

WABERSKI, D.; PETRUNKINA, A. M.; TÖPFERPETERSEN, E. Can external quality control improve pig IA efficiency? Theriogenology, v. 70, n. 8, p. 1346-1351, 2008. 
